\title{
Fuzzy Subgroup Mining for Gene Associations
}

\author{
Marco Ortolani \\ University of Palermo \\ Dept. of Electrical Engineering \\ 90128 Palermo, Italy
}

\author{
Ondine Callan \\ VistaGen Therapeutics, Inc. \\ 1450 Rollins Road \\ Burlingame, CA 94010, USA \\ Michael R. Berthold \\ Dept. of Computer and Information Science \\ Konstanz University \\ Fach M712, 78457 Konstanz, Germany \\ Email: Michael.Berthold@uni-konstanz.de
}

\author{
David E. Patterson \\ Tripos, Inc. \\ 601 Gateway Blvd., Suite 720 \\ South San Francisco, CA 94080, USA
}

\begin{abstract}
When studying the therapeutic efficacy of potential new drugs, it would be much more efficient to use predictors in order to assess their toxicity before going into clinical trials.

One promising line of research has focused on the discovery of sets of candidate gene profiles to be used as toxicity indicators in future drug development. In particular genomic microarrays may be used to analyze the causality relationship between the administration of the drugs and the so called gene expression, a parameter typically used by biologists to measure its influence at gene level. This kind of experiments involves a high throughput analysis of noisy and particularly unreliable data, which makes the application of many data mining techniques very difficult.

In this paper we explore a fuzzy formulation of the a priori algorithm, a technique whose crisp version is commonly used to mine for subgroups in large datasets; the purpose is to extend the original method, already sujitable to deal with large amount of data, in a way that naturally allows the user to deal with the intrinsic imprecision in the data.

The algorithm is tested on real data coming from experimental genomic data.
\end{abstract}

\section{INTRODUCTION}

Genomic arrays are a powerful instrument of analysis for the experts in the field of drug toxicity research, since they provide them with a better global picture and allow them to analyze the interactions among thousands of genes simultaneously.

One important advantage when working with microarrays is thus the high data throughput; on the other hand this implies the need of coupling their use with sophisticated data mining techniques in order to extract the information that is actually useful for the case at hand. Moreover, as in many other real world applications, the original data is usually not reliable enough, because of imprecisions in the measurements, noise or other causes inherent to the nature of the data itself.

In this paper we present a modification of the $a$ priori algorithm, a well known method aimed at finding regularities in a series of data in order to discover co-occurring elements.

The very large amount of possible combinations that need to be analyzed cannot be dealt with through exhaustive search, so obviously particular attention must be paid to the choice of the data structure used to store the information. Also, as already pointed out, the poor quality of the data cannot be ignored.
The fuzzy formulation of the a priori algorithm presented here addresses the latter point.

Section II introduces the basic concept of "association rule" and Section III summarizes the original formulation of the $a$ priori algorithm; Section IV then presents the fuzzy extension. We discuss our results in Section V and finally in Section VI we conclude and discuss possible further extensions.

\section{Association RULES}

The a priori algorithm belongs to a class of methods whose goal is to find regularities in a set of data in order to discover co-occurring elements and to generate a set of rules that can be used for prediction [1], [2].

A field in which it may typically find applications is market basket analysis, where one wants to determine the items that customers buy together so that, knowing what products are already in the shopping cart, it may be possible to infer the presence of other products; such information is stored in the form of an association rule.

An association rule stores the attributes for a particular sample; in the case of market basket analysis, the customers represent the samples, and the items they bought are the respective attributes. In the standard formulation of the problem an association rule contains crisp information (either an item has been bought or not). The goal is, of course, the selection of interesting rules from the whole set, so some measure is required in order to attach a relevance value to each rule.

One of the quantities that are commonly considered is the so called support of the rule, that is the percentage of samples for which the rule applies. Usually a minimum support is specified, so that only the samples represented by those rules whose attributes satisfy at least the specified number of rules are selected (that is, we are interested in frequent subgroups of items.)

Despite not being conceptually too challenging in principle, the task of rule selection presents obvious difficulties, since an exhaustive search through all the rules is practically unfeasible due to the very high number of possible combinations; what is needed is an effective way to store the elements and to process them. 


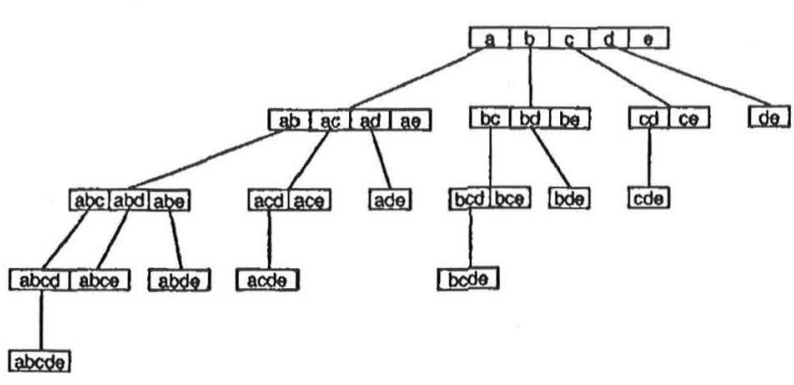

Fig. 1. A prefix tree built using five attributes. The nodes represent the subgroups with their associated counters; when no pruning occurs, the tree grows until no more elements can be added to a subgroup; the ordering of the attributes ensures that duplicate subgroups are avoided.

Many sophisticated algorithms for mining association rules exist in literature [2] and several modifications of the basic formulation of the problem have been proposed in an attempt to improve performance [7], or to consider different definitions for the constraints [5] and so on. We argue that an a prioribased approach combined with use of fuzzy logic may still prove effective, especially when dealing with unreliable data.

\section{THE A PRIORI ALGORITHM}

In this paper we follow the implementation described in [3] and use a prefix tree as the main data structure.

The structure of this tree is sketched in Figure 1. Each node of the tree represents an association rule identified through its attributes; it also needs to contain a counter indicating the frequency of the rule in the original dataset in order to be able to assess whether the support constraint is satisfied or not.

Storing the tree is expensive in terms of memory, and its implementation also affects the performance during the search for frequent subgroups. The idea behind a prefix tree is that we do not need to store the complete information about a rule at each node.

More precisely, each of the nodes at depth $n$ in the tree represents the last element in a rule describing a sample with $n$ attributes and it will also contain the counter. The whole rule can be easily reconstructed and this solution seems also a natural implementation that facilitates later access to the data.

The tree is built incrementally as described by the pseudocode in Figure 2, where we use the term " $n$-frontier" to indicate the collection of all subgroups containing $n$ attributes.

The generic $n$-th iteration consists of two steps: at first all rules containing $n$ attributes are generated; after that, the ones not satisfying the frequency requirement are pruned, thus generating a clean $n$-frontier. The rules of size $n+1$ can be easily built by selecting one element at a time from the 1 -frontier and adding it to one of the subgroups of size $n$. Moreover, since for our purposes two subgroups are identical regardless of how the elements they contain are arranged, we can avoid testing unnecessary combinations if all the elements are assigned an identifier that defines an order on them. Extending a subgroup will only be allowed with elements
// Choose minimum required support $\bar{\sigma}$

// Initialize variables

$n=1 / /$ frontier counter

// Initialize first frontier

$F_{1}=\left\{\left(a_{i}\right) \|\right.$ support $\left.\left(\left(a_{i}\right)\right) \geq \bar{\sigma}\right\}$

// Build remaining frontiers

REPEAT

// Expand each subgroup in previous frontier

$\forall S:=\left\{\left(a_{1}^{S}, a_{2}^{S}, \ldots, a_{n-1}^{S}\right)\right\} \in F_{n-1}:$

/I using elements from the first frontier

$A=\left\{a_{i} \|\left(a_{i}\right) \in F_{1} \wedge\left(\right.\right.$ index $\left.\left(a_{i}\right) \geq \operatorname{index}\left(a_{n-1}^{S}\right)\right\}$

$\forall a \in A: F_{n}=F_{n} \cup(S ; a)$

// clean $n$-frontier

$\vec{F}_{n}=\left\{S_{i} \in F_{n} \|\right.$ support $\left.\left(S_{i}\right)<\vec{\sigma}\right\}$

$F_{n}=F_{n}-\bar{F}_{n}$

// Update frontier counter

$n=n+1$

UNTIL $\left(F_{n}=\{\}\right)$ or (stop condition)

Fig. 2. Pseudo-code describing the process of building the prefix tree.

whose index is greater than the those of the elements already present. Obviously the tree is greatly simplified by eliminating this redundancy.

No element added to a subgroup that does not satisfy the support constraint may possibly generate a frequent subgroup at any successive level thus justifying the early pruning of the tree. Since growing the tree may cause a combinatorial explosion, the pruning mechanism is meant to try and keep the memory consumption low since the frontiers deep in the tree will contain fewer and fewer elements to be further expanded.

This iterative process is repeated until it is no more possible to expand the last frontier or another stop condition is true, as may be the case if a maximum depth for the tree was specified.

Figure 1 shows an example of such prefix tree, with its typical highly unbalanced structure. In this example, the tree was built starting from five attributes that, by themselves, appear in at least as many rules as specified by the chosen support; in other words, those elements will form the 1frontier.

Assuming that all nodes satisfy the constraint for the support at each depth level, no pruning will be required; nevertheless the ordering of the attributes ensures that repeated subgroups are avoided. All subgroups can be easily retrieved from this dạta structure. -.

\section{FUZZY SUBGROUP MINING}

The approach just outlined provides an efficient solution in terms of memory and time requirements. One limitation, though, is that in the original formulation the association rules are meant to represent crisp information (in the market basket analysis example, a customer either bought an item or not).

In many real world applications it is very unlikely that the original data is already accurate enough to be used as an input for the algorithm; for instance, when working with biological data it is mandatory to take into account some imprecision due to measurements or just biological variability, so such crisp decision lines are inappropriate.

Moreover, the generation of the association rules themselves is an intrinsically imprecise task since a clearly cut decision 
// Choose minimum required support $\vec{\sigma}$

// Initialize variables

$n=1 / /$ frontier counter

FOR all chosen fuzzy thresholds $\mu_{0}, \mu_{1}, \ldots, \mu_{k}$

// Re-build first frontier using data obtained with current fuzzy threshold

$F_{1}^{\mu_{k}}=\left\{\left(a_{i}\right) \|\right.$ support $\left.{ }^{\mu_{k}}\left(\left(a_{i}\right)\right) \geq \tilde{\sigma}\right\}$

// Build additional frontiers

REPEAT

If Expand each subgroup in previous frontier

// (previous frontier may have been built during last run)

$\forall S:=\left\{\left(a_{1}^{S}, a_{2}^{S}, \ldots, a_{n-1}^{S}\right)\right\} \in F_{n-1}$ :

If using elements from the first fronitier

$A=\left\{a_{i} \|\left(a_{i}\right) \in F_{1}^{\mu_{k}} \wedge\left(\operatorname{index}\left(a_{i}\right) \geq \operatorname{index}\left(a_{n}{ }_{-1}\right)\right\}\right.$

$\forall a \in A: F_{n}=F_{n} \cup(S ; a)$

I/ clean $n$-frontier

$\vec{F}_{n}=\left\{S_{i} \in F_{n} \| \operatorname{support}\left(S_{i}\right)<\ddot{\sigma}\right\}$

$F_{n}=F_{n}-\ddot{F}_{n}$

// Update frontier counter

$n=n+1$

UNTIL $\left(F_{n}=\{\right.$ ) or (stop condition)

ENDFOR

Fig. 3. Pseudo-code describing the process of building the prefix tree in several nuns each using a different fuzzy threshold.

line is rarely available; on the contrary, it is often desirable to leave some freedom to the users, in terms of interaction with the algorithm.

A natural extension is thus the embedding of fuzzy logic [13] in the algorithm itself by letting the attributes stored in the association rules represent a fuzzy degree of membership. Computing the support for each rule will require the choice of a threshold, which of course will influence the pruning of the prefix tree.

The choice of a fuzzy threshold obviously modifies the starting data; in particular a lower threshold may allow more elements to satisfy the constraint on the support and eventually larger subgroups to be discovered Since in most cases the original data is imprecise, allowing this kind of flexibility may represent a great advantage.

The algorithm is described in the form of pseudo-code in Figure 3 , Besides the additional loop introduced to consider one different fuzzy threshold at a time, the main difference with Figure 2 is in the construction of the first frontier. The notation $F_{1}^{\mu_{k}}$ shows that it is now dependent on the fuzzy threshold, as already explained; in summary more elements are added to the first frontier and only the last frontier is considered as a source for subgroups to expand.

The underlying idea is that, by choosing a maximum allowed number of elements per subgroup, a minimum support and a fuzzy threshold for each iteration; the user is now able to tune the algorithm toward a subset of the whole search space where it is more likely to find useful subgroups

The example in Figure 4 illustrates this point Let us assume that after choosing a fuzzy threshold 0 , five attributes initially satisfy the support constraint (let them be $a, b, c, d$ and $f$ ) As previously mentioned, the attributes are ordered using an internal index.

In this first iteration, the tree is built as described earlier

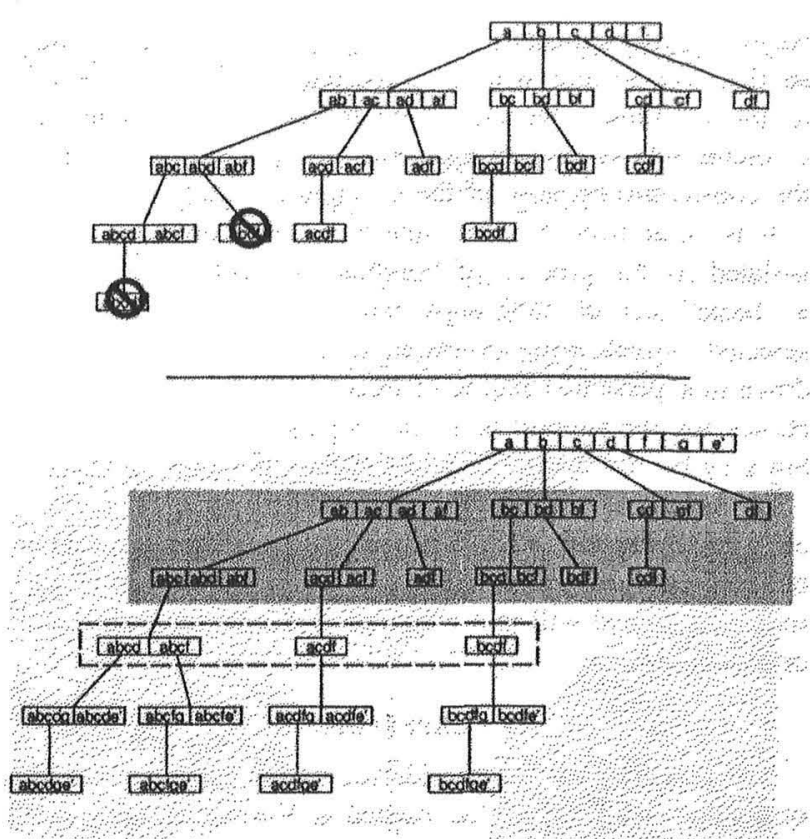

Fig. 4. The evolution of the prefix tree under two successive choices for the fuzay threshold. In the bottom half the dashed area represent the part of the tree that will be expanded, whereas the shadowed area will be "frozen"

and for the sake of the example we assume that two of the discovered subgroups do not satisfy the requirements for the minimum support and are pruned from the tree, as shown in the upper part of Figure 4.

A successive run with a fuzzy threshold $1<0$ discovers another attribute $(\mathrm{g})$ that now satisfies the constraint, moreover attribute $e$ is reconsidered and accepted in the expanded first frontier because, thanks to the lower fuzzy threshold, it now satisfies the support constraint. This attribute is assigned a higher internal index and is appended at the end of the list of available attributes (this is indicated by $e^{\prime}$ in the lower part of Figure 4).

The tree may now continue to grow by expanding the subgroups found in the last frontier; however the process is slightly different than in the original formulation of the algorithm. The shadowed area in the figure shows a part of the tree that was generated by previous runs, the leaves of the tree contained in this area will not be expanded anymore, rather we ignore this part of the search tree and focus only on the last frontier.

Since we are arguably interested in finding larger subgroups this represents a trade-off that allows us to get a deeper insight into what was already found with a more restrictive constraint while keeping the tree small. Comparing the bottom half of Figure 4 with the top one, it is evident that we have succeeded in further expanding the tree (depth-wise) without having to consider possibly less interesting subgroups.

Obviously this technique poses a problem for the ordering of the elements, that we want to maintain homogeneous throughout different runs in order to keep the tree consistent. 
Newly found elements will be assigned a higher internal hdex so that the sorting will be consistent (as in the case of $g$ in the example); we also need to take into account those elements whose support increases (and possibly now satisfies the constraint) because of the ehanged threshold $\left(e^{\prime}\right)$.

It is clear from this example that two main steps may be isolated in the process of building the prefix tree. Initially, a "basie" set of subgroups satisfying the requirements is selected, which corresponds to narrowing the search space down to a particular region of interest. An initial conservative choice for the support helps in keeping the search tree smal but may be too restrictive and overlook potential candidates.

By softening the fuzzy constraints this hard core will be allowed to expand, adding larger subgroups to the intial set newly found subgroups will still satisfy the original support requirement and will in a way represent a "refinement" of the original solution.

\section{EXPERTMENTS}

The research we are considering as the source of our data originated from a study conducted at VistaGen Therapeutics, Ine; the purpose of this study is to investigate the possibility of using gene expression profiles as predictors for potential drug toxicity

\section{A. The data}

The data used in our experiments was generated using gene microarray technology [1]. Microarrays can be regarded as matrices in which each spot contains information about the amount of RNA for a particular sample; the number of spots per array is typically in the order of several thousands.

This kind of data has often presented a challenge to researcher in the field of data mining due to its characteristics of noisiness and unreliability, and many different approaches have been already documented in literature $[4],[6],[8],[10]$

For our purposes it is sufficient to say that, starting from the raw data, it is possible to generate a matrix of intensity values representing RNA expression profiles.

An experiment then consists in measuring the effects produced by a particular drug in terms of variation in the gene expression levels; even though the actual biological path may remain partially unknown, the idea is that a relationships may be observed between the final effects (the gene expression levels) and the cause (the administration of the drug).

Our experiments involved a sel of nephrotoxic drugs tested on two selected kídney cell línes to produce an output consisting in the expression levels of about 8000 genes. Previous tests conducted in laboratories on human renal epithelial cells suggest that they show different sensitivities to different drugs, very much as observed in vivo. In particular the two cell lines considered here are two different areas of the kidney (the Proximal Straight Tubule, or PST, and the Collecting Tubule, or (T), respectively the areas most and least sensitive to treatment:

The object of the present research are genes that are involved in the early response to the drugs, that is before acute
TABLE I

THE EXPERTS' DEFINITIONS FOR FOUR GROUPS OF INTEREST. THEY CAN BE EASILY TRANSLATED INTO FUZZY RULES.

\begin{tabular}{|c|c|c|}
\hline Definition \\
\hline Group 1 & PST fold down AND CT fold dp or tro change \\
\hline Group 2 & PST fold UP AND CT fold down or no change \\
\hline Group 3 & PST fold no change AND CT fold down \\
\hline Group 4 & PST iold no change AND CT fold uo
\end{tabular}

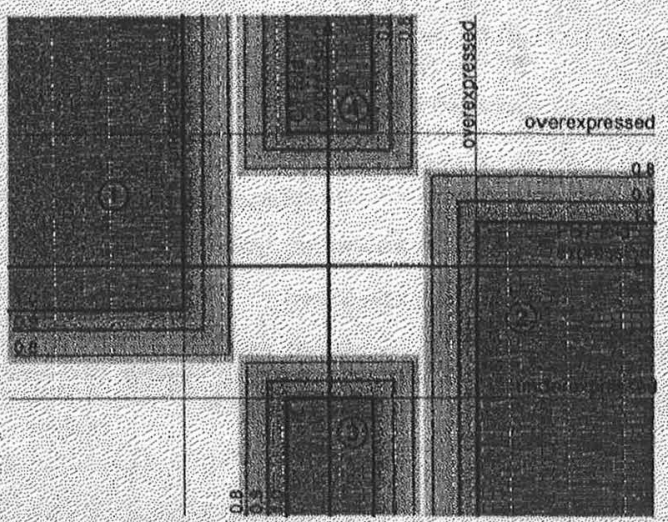

Fig. 5. A graphical representation of the fuzzy groups defined in Table 1. The borders of the fuzzy regions vary depending on the choice of the threshold.

toxicity manifests itself; in order to identify them, an appropriate concentration was selected for five target drugs: Cisplatin, Cyclosporin, Gentamycin, Tobramycin and Kanamyein.

The gene subgroup discovery process is complicated by the fact that the role of most genes is still unknown; indeed one of the aims of the biologists is to find groups of undocumented genes in order to broaden their knowledge of the biological pathways that cause a drug to be toxic.

In a situation like this an exploratory analysis that allows the scientist to get some preliminary results in order to re test, refine them and possibly validate then against existing literature is of much greater use than a static algorithm.

\section{B. Mining for predictors}

From previous studies it is already known to biologists that potentially interesting genes are the ones that show a differential response in the two considered cell lines (PST and CT), so they were asked to formalize a description of the expected targets in terms of groups of interest, which will then be used to define the association rules, or in other words to decide whether a gene is to be considered overall expressed or not for a certain drug.

The quantity that would be natural to consider for this purpose is the bare expression level for a given gene, but unfortunately this is usually not particularly useful per se, since it is influenced by too many factors, including intrinsic biological variability, which basically deteriorate the signal/noise ratio. Biologists prefer to rely on a different quantity, the so called fold expression, which is defined as the ratio of the expression 
TABLE II

EACH ROW SHOWS, FOR THE GIVEN THRESHOLD, HOW MANY GBNES WERE SELECTED, HOW MANY GENES HAD THEIR SUPPORTCHANGED BECAUSE OF THE NEW FUZZY THRESHOUD AND WHAT SUBGROUPS WERE DISCOVBRED. FOR THE LAST COLUMN, THE NOTATION $X(Y)$ INDICATES THAT Y SUBGROUES WERE ROUND CONTAINING X GENES.

\begin{tabular}{|c|c|c|c|}
\hline Group 1, & \multicolumn{3}{|c|}{} \\
\hline Threshold & Found & Changed & Subgroups \\
\hline 1.0 & 45 & & $\begin{array}{c}4(1) 5(1) 6(3) \\
8(1) 17(1)\end{array}$ \\
\hline 0.9 & 43 & 3 & $34(1)$ \\
\hline 0.8 & 48 & 10 & $44(1)$ \\
\hline
\end{tabular}

\begin{tabular}{|c|c|c|c|}
\hline Group 2 & & \\
\hline Threshold & Found & Changed & Subgroups \\
\hline 1.0 & 7 & & $2(1) 5(1)$ \\
\hline 0.9 & 7 & 2 & $6(1)$ \\
\hline 0.8 & 14 & 1 & $9(1)$ \\
\hline
\end{tabular}

\begin{tabular}{|c|c|c|c|}
\hline Group 3 & & \\
\hline Threshiold & Found & Changed & Subgroups \\
\hline 1.0 & & & \\
\hline 0.9 & & & $3(1)$ \\
\hline 0.8 & 4 & &
\end{tabular}

\begin{tabular}{|c|c|c|c|}
\hline Group 4 & \multicolumn{3}{|c|}{} \\
\hline Threshold & Found & Changed & Subgroups \\
\hline 1.0 & 3 & & $2(2)$ \\
\hline 0.9 & 1 & 2 & $4(2)$ \\
\hline 0.8 & 7 & & $6(2)$ \\
\hline
\end{tabular}

levels of genes observed in the sample before (controt) and after (treatment) the drug is administered.

Table I presents four such groups as defined by the bi ologists depending on the relative importance of the gene expression in the two cell lines. For instance, they argue that a decrease in the fold expression in the PST region coupled with a contemporary increase or stability in the CT region is a sign of drug toxicity (as represented by group 1).

Even so, there is no criterion providing an all-purpose cutoff value to decide what values of fold expression correspond to the "expressed" status. A 2 -fold expression cut seemed a reasonable choice for the present data, even though a very large amount of genes would lie very close to either side of this crisp border.

As already pointed out, the binary perspective is probably too restrictive, so we reformulated the definitions of such groups in term of fuzzy rules, which have the advantage of expressing in a better and more intuitive way the imprecise background knowledge provided by the expert

When PST and CT are regarded as linguistic variables, the fuzzy property up corresponds to "over-expressed" and the opposite holds for down. An unnoticeable modification in the value of the fold expression is indicated by no change. The properiles can be combined using the usual fuzzy operators [9], [12].

A graphical representation of the fuzzy groups is given in Figure 5

Table II presents the results produced by our algorithm in this case. For all the groups we required a minimum support
TABLE III

A MODIFIED DEHINITION FOR THE AREAS OF INTEREST, DRFINING TWO BROADER GROUPS,

\begin{tabular}{|c|c|}
\hline & Definition \\
\hline Group 1 & $\begin{array}{l}\text { (PST fold / CT fold) doon } \\
\text { AND } \\
\text { (PST fold down OR CT fold up) }\end{array}$ \\
\hline & $\begin{array}{l}\text { (PST fold / CT fold) up } \\
\text { AND } \\
\text { (PST, fold up or CT, fold down) }\end{array}$ \\
\hline
\end{tabular}

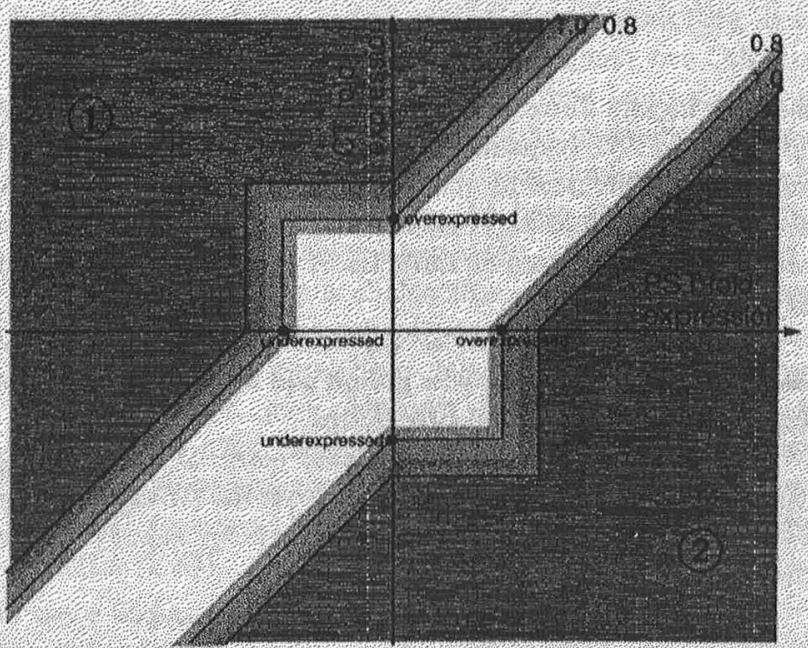

Fig. 6. A graphical representation of the furzy groups defined in Table in Both axes are in logarithmic scale, two choices of the fuzzy threshold show how the borders vary.

of at least four of the drugs. We considered three runs with fuzzy thresholds $0=1.0,1=0.9,2=0.8$ respectively.

Let us consider Group 1 as an example of the way the algorithm works. For $0=1.0$, the largest subgroup found contains 17 genes; the total number of genes that, considered singularly, satisfy the constraint is 45 . There are also several smaller subgroups that differ in at least one gene or for the drugs they cover (for instance, there are three subgroups containing six genes each).

When we lower the threshold to $\quad 1=0.9$, we expand the first frontier to contain 43 new genes. Moreover, three genes that were previously excluded now meet the requirements for the support. This results in the discovery of a new subgroup containing 34 genes.

With $2=0.8 .48$ more genes are allowed; the support is now high enough for six more genes and finally a subgroup of 44 genes is discovered.

Those results were examined by the biologists and compared to what is already known from literature; they then decided to modify the definition of the groups, while maintaining the basic idea, that is selecting genes whose expression levels are noticeably different in PST and CT

In the new definition, only two larger groups were con- 
TABLE IV

SELECTED GENES FROM THE MECHANISM-BASED PREDICTORS

\begin{tabular}{|c|c|c|}
\hline Genes of Interest & Class & Drugs \\
\hline $\begin{array}{l}\text { TNF, LTBR, p21, Cytochrome P450, E2F3, GAP(DH), PDGFA, HSP, } \\
\text { Interleukin, PIK3, STAT, Toll-like receptor, Uncoupling Protein }\end{array}$ & DNA damage & Cisplatin \\
\hline $\begin{array}{l}\text { Caspase 9, STAT, DEFCAP, Calpain 6, multiple HSP, GSTP1, IGFP, } \\
\text { LTB,c-AMP, Cytochrome C }\end{array}$ & Aminoglycosides & $\begin{array}{c}\text { Gentamycin } \\
\text { Tobramycin, Kanamycin }\end{array}$ \\
\hline $\begin{array}{l}\text { CREB, CAPG, CDC, CDK, GADD45, E2F,COX, Cytochrome p450, } \\
\text { EIF,FGF, HSP, HIF, IL6, MAPK8, PPAR }\end{array}$ & $\begin{array}{l}\text { Calcium } \\
\text { transport }\end{array}$ & $\begin{array}{l}\text { Kanamycin } \\
\text { Cyclosporin }\end{array}$ \\
\hline $\begin{array}{l}\text { ARL2, ASB3, Calpain, CDC, CENPE, DEFCAP, Laminin, LTB, } \\
\text { Acetyltransferase, CAMP kinase, SRC phosphoprotein 2, } \\
\text { Cytochrome C, Elongation factor B }\end{array}$ & $\begin{array}{l}\text { Ribosomal } \\
\text { inhibitors }\end{array}$ & $\begin{array}{l}\text { Gentamycin } \\
\text { Tobramycin }\end{array}$ \\
\hline
\end{tabular}

sidered, ideally corresponding to the two cases in which the influence in PST is qualitatively more relevant than in CT and vice versa. This new formulation takes into account not only the value of the fold-expression in PST or CT, but their ratio as well, as is summarized in Table III. A graphical interpretation is given in Figure 6.

Experiments analogous to the ones previously described were carried on using the new groups and we considered genes expressed in either of the two groups.

It is worth noting that in this case some of the resulting subgroups were considered relevant from the biological point of view, since they corresponded to groupings already known from literature.

Biologists defined a list of "classes" that grouped some of the drugs according to their known mechanism of action and examined the subgroups discovered by our algorithm that fell into one of those classes (that is, whose support matched one of the classes).

They noticed that some of the genes belonging to those subgroups had already been described in the literature and were known to be related to that particular mechanism of action, while other genes were a new addition. Those genes appeared to be related to particular drugs, responding to them and signaling their toxicity, thus representing good candidates as predictors for the respective drug class.

Table IV presents a selection of those genes together with the class they describe and the drugs that form this class.

\section{Conclusions}

The use of a fuzzified version of the a priori algorithm proved to be very promising for the application to genomic data mining.

The peculiar nature of the data, namely highly noisy biological data, makes it very difficult to use traditional crisp data analysis tools; our approach, in addition, was considered very intuitive by the experts and allowed them to explore the data and refine their hypotheses, in some cases confirming them, and to select a smaller sample of interesting candidates from the whole dataset.

The results found during the preliminary experiments described here are encouraging, since they are supported by existing literature and also offer new insights that open the way to further research.
Future work will include more testing on larger datasets and will benefit from increased interaction between the experts in the two fields of data analysis and biology.

\section{REFERENCES}

[1] Rakesh Agrawal, Tomasz Imielinski, and Arun N. Swami. Mining association rules between sets of items in large databases. In Peter Buneman and Sushil Jajodia, editors, Proceedings of the 1993 ACM SIGMOD International Conference on Management of Data, pages 207 216, Washington, D.C., 26-28 1993.

[2] Rakesh Agrawal and Ramakrishnan Srikant. Fast algorithms for mining association rules. In Jorge B. Bocca, Matthias Jarke, and Carlo Zaniolo, editors, Proc. 20th Int. Conf. Very Large Data Bases, VLDB, pages $487-$ 499. Morgan Kaufmann, 12-15 1994.

[3] Christian Borgelt and Rudolf Kruse. Induction of association rules: A priori implementation. In Physica Verlag, editor, Proc. of the 15th Conference on Computational Statistics (Compstat 2002, Berlin, Germany), Heidelberg, Germany, 2002.

[4] M. Brown, W. Grundy, D. Lin, N. Cristianini, C. Sugnel T. Furey, M. Jr, and D. Haussler. Knowledge-based analysis of microarray gene expression data by using support vector machines. In Proc. Natl. Acad. Sci., volume 97, pages 262-267, 2000.

[5] Edith Cohen, Mayur Datar, Shinji Fujiwara, Aristides Gionis, Piotr Indyk, Rajeev Motwani, Jeffrey D. Ullman, and Cheng Yang. Finding interesting associations without support pruning. Knowledge and Data Engineering, 13(1):64-78, 2001.

[6] Sandrine Dudoit, Jane Fridlyand, and Terence P. Speed. Comparison of discrimination methods for the classification of tumors using gene expression data. Journal of the American Statistical Association 97(457):77-87, 2002

[7] Christian Hidber. Online association rule mining. In ACM SIGMOD Int. Conf. on Management of Data, pages 145-156, 1999.

[8] P. O. Brown M. B. Eisen, P. T. Spellman and D. Botstein. Cluster analysis and display of genome-wide expression patterns. In Proc. Nath Acad. Sci. USA, volume 95, pages 14863-14868, Dec 1998.

[9] A. D. Nola and A. Ventre, editors. The Mathematics of Fuzzy Systems. TƯV, Rheinland, Köln, 1986.

[10] Donna K. Slonim, Pablo Tamayo, Jill P. Mesirov, Todd R. Golub, and Eric S. Lander. Class prediction and discovery using gene expression data. In RECOMB, pages 263-272, 2000.

[11] Gordon K. Smyth, Yee Hwa Yang, and Terry Speed. Statistical issues in cDNA microarray data analysis. Functional Genomics: Methods and Protocols, 2002.

[12] S. Weber. A general concept of fuzzy connectives, negations and implications based on t-norms and t-conorms. Fuzzy Sets and Systems, 95:113-134, Oct 1983.

[13] Lotfi A. Zadeh. Fuzzy sets. Information and Control, 8:338-353, 1965 